\title{
Novel fluorescent isoquinoline derivatives obtained via Buchwald-Hartwig coupling of isoquinolin-3-amines
}

\author{
József Balog, Zsuzsanna Riedl, György Hajós, ${ }^{*}$ Zsombor Miskolczy, and László Biczók \\ Chemical Research Center, Hungarian Academy of Sciences, Pusztaszeri ut 59-67. H-1025 \\ Budapest, Hungary \\ E-mail:ghajos@chemres.hu
}

Dedicated to Prof. Ferenc Fülöp on the occasion of his 60th birthday

\begin{abstract}
Isoquinolin-3-amines bearing an alkyl group or hydrogen atom in position 4 easily underwent Buchwald-Hartwig coupling reactions with various substituted aryl halides. Investigation of the selected new derivatives with fluorescent spectroscopy revealed that the reaction products have similar photophysical properties to those of isoquinolin-3-amine except the $N$-(nitrophenyl) derivatives, which emit negligible fluorescence.
\end{abstract}

Keywords: Isoquinolinamine, pyridinamine, fluorescence, Buchwald-Hartwig amination

\section{Introduction}

Recently we have published ${ }^{1}$ that some isoquinoline derivatives bearing a morpholine group in position 1 or 3 represent a new group of fluorescent compounds. In the course of continuation of our activity in the area of isoquinolinamines, synthesis of variously substituted $\mathrm{N}$ arylisoquinolinamines has been decided. Such compounds could serve as valuable starting compounds for subsequent ring closure reactions. To this end, use of the available isoquinolin-3amine as well its 4-methyl and 4-benzyl derivative in Buchwald-Hartwig amination ${ }^{2-4}$ of aryl halides has been envisaged.

\section{Results and Discussion}

Inspection of the pertinent literature revealed that very few cases of direct amination with isoquinolinamine have been carried out. ${ }^{5}$ Similarly, Buchwald-Hartwig amination with 
quinolinamines and pyridinamines and other related $N$-heterocycles have also been published by the same research group. ${ }^{6}$

In the frame of the present study, unsubstituted and 4-alkyl substituted isoquinolin-3-amines $\left(\mathbf{1}, \mathrm{R}^{4}=\mathrm{H}, \mathrm{CH}_{3}\right.$ and $\mathrm{CH}_{2} \mathrm{C}_{6} \mathrm{H}_{5}$, respectively) were treated with various substituted bromobenzene derivatives and 2,3-dibromopyridine as shown in Scheme 1. The substituents of the bromobenzene reagent involved $m$ - and $p-\mathrm{Cl}, o-, m-$, and $p-\mathrm{Br}, o-$ and $p-\mathrm{NO}_{2}, o-, m-$, and $p$ COOEt, $o$-CHO moieties. As a ligand to $\mathrm{Pd}_{2}(\mathrm{dba})_{3}$ JohnPhos and Xantphos provided the best results. As bases, cesium carbonate and sodium $t$-butoxide were used. The products have been obtained in $46-94 \%$ yields. The derivatives and experimental conditions are summarized in Table 1.

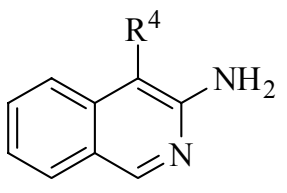

1

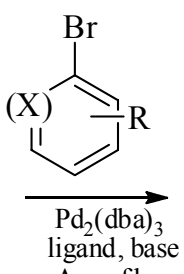

Ar, reflux<smiles>[R]c1ccccc1Nc1ncc2ccccc2c1[R4]</smiles>

2

\section{Scheme 1}

In order to reveal the photophysical properties of the new compounds, some selected derivatives have been studied by absorption and fluorescence spectroscopy. As shown in Figure 1, the nitro-substitution leads to a significant bathochromic shift of the first absorption band, whereas the introduction of the $p-\mathrm{COOC}_{2} \mathrm{H}_{5}$ moiety brings about marked blue- and red-shifts for the first and second absorption bands, respectively. The $m-\mathrm{COOC}_{2} \mathrm{H}_{5}$ and $p-\mathrm{Cl}$ substituents have smaller effect. Intramolecular hydrogen bonding between $\mathrm{N}-\mathrm{H}$ and the oxygen of the $o-\mathrm{NO}_{2}$ may contribute to the considerable diminution of the energy of the lowest singlet-excited state in the case of 2l. Table 2 summarizes the photophysical parameters of the new compounds and, for the sake of comparison, those of isoquinolin-3-amine $\left(\mathbf{1}, \mathrm{R}^{4}=\mathrm{H}\right)$. The fluorescence maximum $\left(\lambda_{\max }(\mathrm{fl})\right)$ exhibits a marked displacement to longer wavelength upon attachment of $\mathrm{N}$-phenyl group to 1a, but $\lambda_{\max }(\mathrm{fl})$ diminishes in the series of $\mathbf{2 a}>\mathbf{2 g}>\mathbf{2 s}>\mathbf{2 t}>\mathbf{1 a}$. The Stokes-shift of the fluorescence band is larger when a substituted phenyl group is attached to the isoquinolinamine skeleton indicating that more substantial structural alteration occurs upon excitation than in the case of 1a. The $N$-(nitrophenyl) derivatives (2o and 2I) emit negligible fluorescence due probably to the enhanced charge transfer character of the lowest singlet-excited state. The new compounds have slightly smaller fluorescence quantum yields $\left(\Phi_{\mathrm{f}}\right)$ and somewhat longer fluorescence lifetimes $\left(\tau_{\mathrm{f}}\right)$ than $\mathbf{1 a},{ }^{1}$ but neither of these quantities is sensitive to the $\mathrm{COOC}_{2} \mathrm{H}_{5}$ or $\mathrm{Cl}$ substitution. The rate constant of fluorescence emission $\left(\mathrm{k}_{\mathrm{f}}\right)$ and radiationless deactivation $\left(\mathrm{k}_{\mathrm{nr}}\right)$ from the singlet excited state were calculated using $\mathrm{k}_{\mathrm{f}}=\Phi_{\mathrm{f}} / \tau_{\mathrm{f}}$ and $\mathrm{k}_{\mathrm{nr}}=$ $\left(1-\Phi_{\mathrm{f}}\right) / \tau_{\mathrm{f}}$ relationships, respectively. As seen in Table 2, practically constant $\mathrm{k}_{\mathrm{nr}}$ values were 
obtained for all fluorescent derivatives, whereas $\mathrm{k}_{\mathrm{f}}$ is slightly larger for $\mathbf{1 a}$ compared to the corresponding values of the phenylamino derivatives.

Table 1. Various derivatives of (2) obtained by Buchwald-Hartwig coupling of isoquinolin-3amines (1)

\begin{tabular}{|c|c|c|c|c|c|c|c|c|c|}
\hline $\mathrm{X}$ & $\mathrm{R}^{4}$ & $\mathrm{R}$ & Ligand & Base & Solvent & $\mathrm{mp}\left({ }^{\circ} \mathrm{C}\right)$ & colour & $\begin{array}{c}\text { yield } \\
(\%)\end{array}$ & $\begin{array}{l}\text { time } \\
\text { (h) }\end{array}$ \\
\hline a $\mathrm{CH}$ & $\mathrm{H}$ & $\mathrm{H}$ & JohnPhos & $\mathrm{NaO}^{t} \mathrm{Bu}$ & Toluene & $101-102$ & yellow & 87 & 5 \\
\hline b $\mathrm{CH}$ & $\mathrm{Me}$ & $\mathrm{H}$ & JohnPhos & $\mathrm{NaO}^{t} \mathrm{Bu}$ & Toluene & $76-78$ & brownish yellow & 77 & 5 \\
\hline c $\mathrm{CH}$ & Benzyl & $\mathrm{H}$ & JohnPhos & $\mathrm{NaO}^{t} \mathrm{Bu}$ & Toluene & $155-157$ & dark yellow & 70 & 5 \\
\hline d $\mathrm{CH}$ & $\mathrm{H}$ & $m-\mathrm{Cl}$ & JohnPhos & $\mathrm{NaO}^{t} \mathrm{Bu}$ & Toluene & $123-126$ & yellow & 91 & 6 \\
\hline e $\mathrm{CH}$ & $\mathrm{Me}$ & $m-\mathrm{Cl}$ & JohnPhos & $\mathrm{NaO}^{t} \mathrm{Bu}$ & Toluene & $77-79$ & pale yellow & 75 & 6 \\
\hline f $\mathrm{CH}$ & Benzyl & $m-\mathrm{Cl}$ & JohnPhos & $\mathrm{NaO}^{t} \mathrm{Bu}$ & Toluene & $72-74$ & pale yellow & 81 & 6 \\
\hline g $\mathrm{CH}$ & $\mathrm{H}$ & $p-\mathrm{Cl}$ & XantPhos & $\mathrm{Cs}_{2} \mathrm{CO}_{2}$ & 1,4-Dioxane & $148-151$ & yellow & 70 & 6 \\
\hline h $\mathrm{CH}$ & $\mathrm{H}$ & $o-\mathrm{Br}$ & XantPhos & $\mathrm{Cs}_{2} \mathrm{CO}_{3}$ & 1,4-Dioxane & $100-102$ & green & 47 & 8 \\
\hline i $\mathrm{CH}$ & $\mathrm{H}$ & $m-\mathrm{Br}$ & JohnPhos & $\mathrm{NaO}^{t} \mathrm{Bu}$ & Toluene & $123-125$ & greenish yellow & 46 & 1 \\
\hline j $\mathrm{CH}$ & Benzyl & $m-\mathrm{Br}$ & JohnPhos & $\mathrm{NaO}^{t} \mathrm{Bu}$ & Toluene & $60-62$ & yellow & 49 & 1 \\
\hline $\mathbf{k} \mathrm{CH}$ & $\mathrm{H}$ & $p-\mathrm{Br}$ & XantPhos & $\mathrm{Cs}_{2} \mathrm{CO}_{2}$ & 1,4-Dioxane & $159-162$ & yellow & 80 & 8 \\
\hline l $\mathrm{CH}$ & $\mathrm{H}$ & $o-\mathrm{NO}_{2}$ & XantPhos & $\mathrm{Cs}_{2} \mathrm{CO}_{3}$ & 1,4-Dioxane & $148-149$ & dark orange & 94 & 5 \\
\hline m CH & $\mathrm{Me}$ & $o-\mathrm{NO}_{2}$ & XantPhos & $\mathrm{Cs}_{2} \mathrm{CO}_{4}$ & 1,4-Dioxane & $130-133$ & dark orange & 94 & 5 \\
\hline n $\mathrm{CH}$ & Benzyl & $o-\mathrm{NO}_{2}$ & XantPhos & $\mathrm{Cs}_{2} \mathrm{CO}_{3}$ & 1,4-Dioxane & $143-146$ & orange & 95 & 5 \\
\hline o $\mathrm{CH}$ & $\mathrm{H}$ & $p-\mathrm{NO}_{2}$ & XantPhos & $\mathrm{Cs}_{2} \mathrm{CO}_{3}$ & 1,4-Dioxane & $174-176$ & brownish yellow & 55 & 4 \\
\hline p $\mathrm{CH}$ & $\mathrm{Me}$ & $p-\mathrm{NO}_{2}$ & JohnPhos & $\mathrm{NaO}^{t} \mathrm{Bu}$ & Toluene & 196-199 & brownish orange & 60 & 2 \\
\hline $\mathbf{q} \mathrm{CH}$ & Benzyl & $p-\mathrm{NO}_{2}$ & JohnPhos & $\mathrm{NaO}^{t} \mathrm{Bu}$ & Toluene & $120-122$ & brownish yellow & 64 & 2 \\
\hline r $\mathrm{CH}$ & $\mathrm{H}$ & $o$-COOEt & XantPhos & $\mathrm{Cs}_{2} \mathrm{CO}_{2}$ & 1,4-Dioxane & $44-46$ & green & 43 & 6 \\
\hline s $\mathrm{CH}$ & $\mathrm{H}$ & $m$-COOEt & XantPhos & $\mathrm{Cs}_{2} \mathrm{CO}_{3}$ & 1,4-Dioxane & $107-109$ & yellow & 84 & 6 \\
\hline t $\mathrm{CH}$ & $\mathrm{H}$ & $p$-COOEt & XantPhos & $\mathrm{Cs}_{2} \mathrm{CO}_{3}$ & 1,4-Dioxane & $141-144$ & yellow & 83 & 6 \\
\hline u $\mathrm{CH}$ & $\mathrm{H}$ & $o-\mathrm{CHO}$ & XantPhos & $\mathrm{Cs}_{2} \mathrm{CO}_{3}$ & 1,4-Dioxane & $92-95$ & brownish yellow & 84 & 2 \\
\hline $\mathrm{N}$ & $\mathrm{H}$ & - & XantPhos & $\mathrm{Cs}_{2} \mathrm{CO}_{3}$ & 1,4-Dioxane & $107-110$ & brownish yellow & 80 & 10 \\
\hline $\mathbf{w} \mathrm{N}$ & Benzyl & - & XantPhos & $\mathrm{Cs}_{2} \mathrm{CO}_{3}$ & 1,4-Dioxane & $116-118$ & brownish yellow & 70 & 10 \\
\hline
\end{tabular}




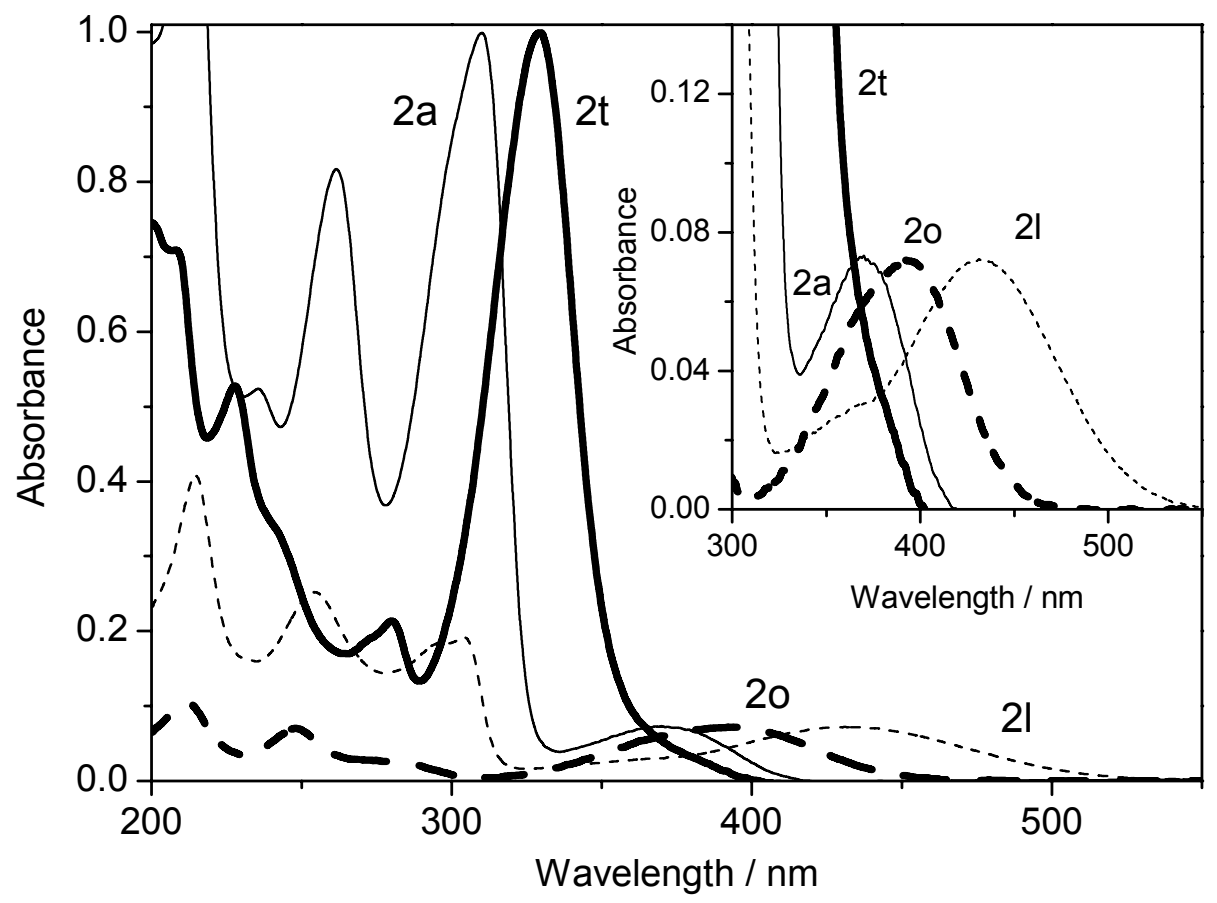

Figure 1. Absorption spectra of 2a (thin line), 2t (thick line), 20 (thick dash line) and 2l (thin dash line) in acetonitrile. Inset shows the zoomed view of the long wavelength range.

Table 2. Photophysical properties of the selected isoquinolin-3-amines in acetonitrile

\begin{tabular}{cccccccc}
\hline & $\mathbf{1 a}^{\mathrm{a}}$ & $\mathbf{2 a}$ & $\mathbf{2 t}$ & $\mathbf{2 s}$ & $\mathbf{2 g}$ & $\mathbf{2 0}$ & $\mathbf{2 l}$ \\
\hline$\lambda_{\max }(\mathrm{abs}) / \mathrm{nm}^{\mathrm{b}}$ & 363 & 371 & $\mathrm{c}$ & $363^{\mathrm{c}}$ & 367 & 395 & 433 \\
$\lambda_{\max }(\mathrm{fl}) / \mathrm{nm}$ & 431 & 464 & 441 & 446 & 457 & $\mathrm{~d}$ & $\mathrm{~d}$ \\
$\Phi_{\mathrm{f}}$ & 0.28 & 0.20 & 0.23 & 0.23 & 0.20 & $<10^{-4}$ & $<10^{-4}$ \\
$\tau_{\mathrm{f}} / \mathrm{ns}$ & 12.4 & 14.2 & 13.0 & 13.3 & 14.2 & $\mathrm{~d}$ & $\mathrm{~d}$ \\
$\mathrm{k}_{\mathrm{f}} / 10^{7} \mathrm{~s}^{-1}$ & 2.3 & 1.4 & 1.7 & 1.7 & 1.4 & $\mathrm{~d}$ & $\mathrm{~d}$ \\
$\mathrm{k}_{\mathrm{nr}} / 10^{7} \mathrm{~s}^{-1}$ & 5.8 & 5.6 & 5.9 & 5.8 & 5.6 & $\mathrm{~d}$ & $\mathrm{~d}$ \\
\hline
\end{tabular}

${ }^{\mathrm{a}}$ Reference $1 .{ }^{\mathrm{b}}$ The location of the maximum of the lowest-energy absorption band, ${ }^{\mathrm{c}}$ shoulder, ${ }^{\mathrm{d}}$ fluorescence is too weak to determine precisely.

\section{Conclusions}

The present results reveal that a great number of actively fluorescent phenylamino and pyridylaminoisoquinolines can be easily obtained by one single manipulation starting from isoquinolinamines in medium to excellent yields. The quantum yield and lifetime of the fluorescence are insensitive to the substituent of the $N$-phenyl moiety but introduction of nitro group makes the compound practically nonfluorescent. 


\section{Experimental Section}

General. Melting points were determined on a Büchi apparatus. The IR spectra were recorded on a Thermo Nicolet Avatar 320 FT-IR spectrometer. NMR measurements were performed on Varian INOVA-300 spectrometer equipped with a $5 \mathrm{~mm}$ inverse detection z-gradient probe. ${ }^{1} \mathrm{H}$ and ${ }^{13} \mathrm{C}$ NMR spectra were measured at room temperature $\left(25^{\circ} \mathrm{C}\right)$ in an appropriate solvent. ${ }^{1} \mathrm{H}$ and ${ }^{13} \mathrm{C}$ chemical shifts are expressed in ppm $(\delta)$ referenced to TMS or residual solvent signals. The elemental analysis has been carried out with an Elementar Vario EL III apparatus (at the Analytical Laboratory for Organic Chemistry, Chemical Research Center, Hungarian Academy of Sciences, H-1025 Budapest, Pusztaszeri út 59-67). Reactions were monitored with Merck silica gel $60 \mathrm{~F}_{254}$ tlc plates $(0.25 \mathrm{~mm}$ thickness $)$. All the chemicals and solvents were used as supplied. The UV-visible absorption spectra were recorded on a Unicam UV 500 spectrophotometer. Corrected fluorescence spectra were obtained on a Jobin-Yvon Fluoromax-P photoncounting spectrofluorometer. Fluorescence lifetimes were measured with time-correlated single-photon counting technique as described previously. ${ }^{7}$ Fluorescence quantum yield was determined relative to that of quinine sulfate in $1 \mathrm{~N} \mathrm{H}_{2} \mathrm{SO}_{4}$, for which a reference yield of $\Phi_{\mathrm{f}}=$ 0.546 was taken. ${ }^{8}$

\section{General procedure for the synthesis of $\boldsymbol{N}$-arylisoquinolin-3-amines}

A round-bottomed flask was charged with $\mathrm{Pd}_{2}(\mathrm{dba})_{3}(5 \mathrm{~mol} \%)$, ligand (10 mol\%), aryl halide (1 $\mathrm{mmol})$, appropriate isoquinolinamine $(1 \mathrm{mmol})$, base $(1.5 \mathrm{mmol})$ and dry solvent $(5 \mathrm{~mL})$. The flask was flushed with argon for $5 \mathrm{~min}$. The mixture was heated at reflux under magnetic stirring. After cooling down to room temperature, the reaction mixture was concentrated and the residue was purified by flash column chromatography on silica gel.

$\mathrm{N}$-Phenylisoquinolin-3-amine (2a). Reaction time $5 \mathrm{~h}$. Eluent: Hexane /EtOAc (4:1), yield: $87 \%$; yellow crystals; $\mathrm{mp} 101-102{ }^{\circ} \mathrm{C} ; v_{\max }\left(\mathrm{KBr}, \mathrm{cm}^{-1}\right): 3239,3054,1631,1453,741 ; \delta_{\mathrm{H}}(300$ $\left.\mathrm{MHz}, \mathrm{CDCl}_{3}\right): 6.91(1 \mathrm{H}, \mathrm{s}, \mathrm{NH}), 7.08\left(1 \mathrm{H}, \mathrm{dd}, J=6.6 \mathrm{~Hz}, 6.4 \mathrm{~Hz}, \mathrm{H}-4^{\prime}\right), 7.20(1 \mathrm{H}, \mathrm{s}, \mathrm{H}-4), 7.27-$ $7.41\left(5 \mathrm{H}, \mathrm{m}, \mathrm{H}-7, \mathrm{H}-2^{\prime}, \mathrm{H}-3^{\prime}, \mathrm{H}-5^{\prime}, \mathrm{H}-6^{\prime}\right), 7.49-7.58(2 \mathrm{H}, \mathrm{m}, \mathrm{H}-5, \mathrm{H}-6), 7.81(1 \mathrm{H}, \mathrm{d}, J=8.2 \mathrm{~Hz}$, $\mathrm{H}-8), 8.96(1 \mathrm{H}, \mathrm{s}, \mathrm{H}-1)$; $\delta_{\mathrm{C}}\left(75 \mathrm{MHz}, \mathrm{CDCl}_{3}\right): 99.3$ (C-4), 120.2 (C-2', C-6'), 122.9 (C-4'), 123.9 (C-7), 124.8 (C-8a), 125.5 (C-5), 128.0 (C-8), $129.7\left(\mathrm{C}-3^{\prime}, \mathrm{C}-5^{\prime}\right), 130.8$ (C-6), 138.8 (C-4a), 141.3 (C-1'), 152.3 (C-3, C-1). Anal. Calcd for $\mathrm{C}_{15} \mathrm{H}_{12} \mathrm{~N}_{2}$ (220.26): C, 81.79; H, 5.49; N, 12.72\% . Found: C, 81.41; H, 5.56; N, 13.03\%.

4-Methyl- $N$-phenylisoquinolin-3-amine (2b). Reaction time 5 h. Eluent: Hexane /EtOAc (4:1); yield: $77 \%$; brownish yellow crystals; $\mathrm{mp} 76-78{ }^{\circ} \mathrm{C} ; v_{\max }\left(\mathrm{KBr}, \mathrm{cm}^{-1}\right)$ : 3253, 1581, 1498, 1247, 746; $\delta_{\mathrm{H}}\left(300 \mathrm{MHz}, \mathrm{CDCl}_{3}\right): 2.47\left(3 \mathrm{H}, \mathrm{s}, \mathrm{CH}_{3}\right), 6.24(1 \mathrm{H}, \mathrm{s}, \mathrm{NH}), 6.95(1 \mathrm{H} . \mathrm{dd}, J=7.0,7.1 \mathrm{~Hz}$, H-4'), 7.21-7.28 (4H, m, H-2', H-3', H-5', H-6'), $7.38(1 \mathrm{H}, \mathrm{dd}, J=7.4,7.7 \mathrm{~Hz}, \mathrm{H}-7), 7.62(1 \mathrm{H}$, dd, $J=7.3,8.13 \mathrm{~Hz}, \mathrm{H}-6)$ ) 7.85-7.90 (2H, m, H-5, H-8), $8.93(1 \mathrm{H}, \mathrm{s}, \mathrm{H}-1)$; $\delta_{\mathrm{C}}\left(75 \mathrm{MHz}, \mathrm{CDCl}_{3}\right)$ : $12.6\left(\mathrm{CH}_{3}\right), 111.2(\mathrm{C}-4), 118.3$ (C-2', C-6'), $121.3(\mathrm{C}-5), 122.6$ (C-4'), 124.1 (C-7), $125.5(\mathrm{C}-8 \mathrm{a})$, 128.6 (C-8), 129.2 (C-3', C-5'), 130.4 (C-6), 137.6 (C-4a), 142.9 (C-1'), 148.9 (C-3), 149.6 (C- 
1). Anal. Calcd for $\mathrm{C}_{16} \mathrm{H}_{14} \mathrm{~N}_{2}$ (234.29): $\mathrm{C}, 82.02 ; \mathrm{H}, 6.02 ; \mathrm{N}, 11.96 \%$. Found: $\mathrm{C}, 81.86 ; \mathrm{H}, 6.14$; $\mathrm{N}, 12.00 \%$.

4-Benzyl- $N$-phenylisoquinolin-3-amine (2c). Reaction time 5 h. Eluent: Hexane /EtOAc (4:1); yield: $70 \%$; dark yellow crystals; $\mathrm{mp} 155-157^{\circ} \mathrm{C} ; v_{\max }\left(\mathrm{KBr}, \mathrm{cm}^{-1}\right)$ : 3414, 1597, 1494, 1315, 747; $\delta_{\mathrm{H}}\left(300 \mathrm{MHz}, \mathrm{CDCl}_{3}\right): 4.39\left(2 \mathrm{H}, \mathrm{s}, \mathrm{CH}_{2}\right), 6.17(1 \mathrm{H}, \mathrm{s}, \mathrm{NH}), 6.95\left(1 \mathrm{H}, \mathrm{dd}, J=6.3,6.2 \mathrm{~Hz}, \mathrm{H}-4^{\prime}\right)$, 7.19-7.39 (10H, m, H-5, H2', H-3', H-5', H-6', H-2", H-3", H-4", H-5", H-6"), 7.61 (1H, dd, $J=$ 7.6, 7.4 Hz, H-6), 7.87-7.91 (2H, m, H-8, H-5 ), $9.03(1 \mathrm{H}, \mathrm{s}, \mathrm{H}-1)$; $\delta_{\mathrm{C}}\left(75 \mathrm{MHz}, \mathrm{CDCl}_{3}\right): 32.1$ $\left(\mathrm{CH}_{2}\right), 112.0$ (C-4), 118.9 (C-2', C-6'), 121.6 (C-5), 122.4 (C-4'), 123.9 (C-7), 125.4 (C-8a), 127.1 (C-4"), 128.4 (C-2', C-6"), 128.8 (C-8), 129.2 (C-3", C-5"), 129.3 (C-3', C-5'), 130.9 (C6), 137.7 (C-4a), $138.6\left(\mathrm{C}-1^{\prime \prime}\right), 142.1$ (C-1'), 149.8 (C-3), 150.5 (C-1). Anal. Calcd for $\mathrm{C}_{22} \mathrm{H}_{18} \mathrm{~N}_{2}$ (310.39): C, 85.13; H, 5.85; N, 9.03\%. Found: C, 85.45; H, 5.90; N, 8.65\%.

$\boldsymbol{N}$-(3-Chlorophenyl)isoquinolin-3-amine (2d). Reaction time 6 h. Eluent: Hexane /EtOAc (3:1); yield: $91 \%$; yellow crystals; $\mathrm{mp} 123-126{ }^{\circ} \mathrm{C} ; v_{\max }\left(\mathrm{KBr}, \mathrm{cm}^{-1}\right): 3259,3057,1630,1589,750 ; \delta_{\mathrm{H}}$ $\left(300 \mathrm{MHz}, \mathrm{CDCl}_{3}\right): 6.84(1 \mathrm{H}, \mathrm{s}, \mathrm{NH}), 7.00\left(1 \mathrm{H}, \mathrm{d}, J=7.7 \mathrm{~Hz}, \mathrm{H}-4^{\prime}\right), 7.18-7.40(5 \mathrm{H}, \mathrm{m}, \mathrm{H}-4, \mathrm{H}-$ 7, H-2', H-5', H-6'), 7.53-7.63 (2H, m, H-6, H-8), 7.83 (1H, d, J = 8.2 Hz, H-5), 8.97 (1H, s, H1); $\delta_{\mathrm{C}}\left(75 \mathrm{MHz}, \mathrm{CDCl}_{3}\right): 100.5$ (C-4), 117.5 (C-6'), $119.2\left(\mathrm{C}-2^{\prime}\right), 122.4$ (C-4'), 124.4 (C-7), 125.1 (C-5), 125.6 (C-8a), 127.9 (C-8), 130.6 (C-5'), 130.9 (C-6), 135.2 (C-3'), 138.7 (C-4a), 142.7 (C1'), 151.2 (C-3), 152.3 (C-1). Anal. Calcd for $\mathrm{C}_{15} \mathrm{H}_{11} \mathrm{ClN}_{2}$ (245.71): C, 70.73; H, 4.35; N, $11.00 \%$. Found: $\mathrm{C}, 70.45 ; \mathrm{H}, 4.51 ; \mathrm{N}, 11.15 \%$.

$\mathrm{N}$-(3-Chlorophenyl)-4-methylisoquinolin-3-amine (2e). Reaction time $6 \mathrm{~h}$. Eluent: Hexane /EtOAc (3:1); yield: $75 \%$; pale yellow crystals; $\mathrm{mp} 77-79{ }^{\circ} \mathrm{C} ; v_{\max }\left(\mathrm{KBr}, \mathrm{cm}^{-1}\right): 3215,2992$, $1572,1475,683 ; \delta_{\mathrm{H}}\left(300 \mathrm{MHz}, \mathrm{CDCl}_{3}\right): 2.48\left(3 \mathrm{H}, \mathrm{s}, \mathrm{CH}_{3}\right): 6.26(1 \mathrm{H}, \mathrm{s}, \mathrm{NH}), 6.89(1 \mathrm{H}, \mathrm{d}, J=7.9$ Hz, H-4') $7.06\left(1 \mathrm{H}, \mathrm{d}, J=8.1 \mathrm{~Hz}, \mathrm{H}-6^{\prime}\right), 7.18\left(1 \mathrm{H}, \mathrm{dd}, J=8.0,8.0 \mathrm{~Hz}, \mathrm{H}-5^{\prime}\right), 7.27$ (1H, s, H-2'), $7.42(1 \mathrm{H}, \mathrm{dd}, J=7.6,7.7 \mathrm{~Hz}, \mathrm{H}-7), 7.65(1 \mathrm{H}, \mathrm{dd}, J=7.1,8.2 \mathrm{~Hz}, \mathrm{H}-6), 7.89(2 \mathrm{H}, \mathrm{m}, \mathrm{H}-5, \mathrm{H}-8)$, $8.95(1 \mathrm{H}, \mathrm{s}, \mathrm{H}-1)$; $\delta_{\mathrm{C}}\left(75 \mathrm{MHz}, \mathrm{CDCl}_{3}\right): 12.6\left(\mathrm{CH}_{3}\right), 112.1(\mathrm{C}-4), 116.1\left(\mathrm{C}-6^{\prime}\right), 117.7\left(\mathrm{C}-2^{\prime}\right)$, 121.0 (C-4'), 122.7 (C-5), 124.6 (C-7), 125.8 (C-8a), 128.6 (C-8), 130.2 (C-5'), 130.6 (C-6), 134.9 (C-3'), 137.5 (C-4a), 144.3 (C-1'), 148.1 (C-3), 149.7 (C-1). Anal. Calcd for $\mathrm{C}_{16} \mathrm{H}_{13} \mathrm{ClN}_{2}$ (268.74): C, 71.51; H, 4.88; N, 10.42\%. Found: C,71.26; H, 4.67; N, 10.65\%.

4-Benzyl- $\mathrm{N}$-(3-chlorophenyl)isoquinolin-3-amine (2f). Reaction time $6 \mathrm{~h}$. Eluent: Hexane /EtOAc (5:1); yield:81\%; pale yellow crystals; $\mathrm{mp} 71-74{ }^{\circ} \mathrm{C} ; v_{\max }\left(\mathrm{KBr}, \mathrm{cm}^{-1}\right): 3224,1591,1488$, 1308,$752 ; \delta_{\mathrm{H}}\left(300 \mathrm{MHz}, \mathrm{CDCl}_{3}\right): 4.39\left(2 \mathrm{H}, \mathrm{s}, \mathrm{CH}_{2}\right), 6.19(1 \mathrm{H}, \mathrm{s}, \mathrm{NH}), 6.89(1 \mathrm{H}, \mathrm{d}, J=7.8 \mathrm{~Hz}$, H-4'), 7.04 (1H, d, J = 8.2 Hz, H-6'), 7.13-7.34 ( 7H, m, H-2', H-5', H-2", H-3", H-4", H-5", H$\left.6^{\prime \prime}\right), 7.42(1 \mathrm{H}, \mathrm{dd}, J=7.6,7.6 \mathrm{~Hz}, \mathrm{H}-7), 7.63(1 \mathrm{H}, \mathrm{dd}, J=8.4,7.1 \mathrm{~Hz}, \mathrm{H}-6), 7.91-7.95(2 \mathrm{H}, \mathrm{m}, \mathrm{H}-$ 5, H-8), $9.04(1 \mathrm{H}, \mathrm{s}, \mathrm{H}-1)$; $\delta_{\mathrm{C}}\left(75 \mathrm{MHz}, \mathrm{CDCl}_{3}\right): 32.1\left(\mathrm{CH}_{2}\right), 112.9(\mathrm{C}-4), 116.6\left(\mathrm{C}-66^{\prime}\right), 118.4(\mathrm{C}-$ 2'), 121.3 (C-4'), 122.5 (C-5), 124.4 (C-7), 125.7 (C-8a), 127.2 (C-4"), 128.3 (C-2", C-6"), 128.8 (C-8), 129.4 (C-3", C-5"), 130.0 (C-5'), 131.1 (C-6), 134.8 (C-3'), 137.7 (C-4a), 138.4 (C-1"), 143.5 (C-1'), 149.1 (C-3), 150.5 (C-1). Anal. Calcd for $\mathrm{C}_{22} \mathrm{H}_{17} \mathrm{ClN}_{2}$ (344.83): C, 76.63; H, 4.97; N, 8.12\%. Found: C, 76. 52; H, 5.21; N: 8.01\%.

$\mathrm{N}$-(4-Chlorophenyl)isoquinolin-3-amine (2g). Reaction time $6 \mathrm{~h}$. Eluent: Hexane /EtOAc (4:1); yield: $70 \%$, yellow crystals, $\mathrm{mp} 148-151^{\circ} \mathrm{C}$; $v_{\max }\left(\mathrm{KBr}, \mathrm{cm}^{-1}\right): 3269,3052,1589,1362,823 ; \delta_{\mathrm{H}}$ 
$\left(300 \mathrm{MHz}, \mathrm{CDCl}_{3}\right)$ : 6.79 (1H, s, NH), $7.12(1 \mathrm{H}, \mathrm{m}, \mathrm{H}-7), 7.22-7.36$ (5H, m, H-2',H-3', H-5', H6', H-4), 7.53-7.60 (2H, m, H-6, H-5), $7.82(1 \mathrm{H}, \mathrm{d}, J=7.0 \mathrm{~Hz}, \mathrm{H}-8), 8.95(1 \mathrm{H}, \mathrm{s}, \mathrm{H}-1)$; $\delta_{\mathrm{C}}(75$ $\mathrm{MHz}, \mathrm{CDCl}_{3}$ ): 99.8 (C-4), 121.2 (C-2', C-6'), 124.2 (C-7), 124.9 (C-8a), 125.4(C-5), 127.5 (C4'), 127.9 (C-8), 129.6 (C-3',C-5'), 130.9 (C-6), 138.7 (C-1'), 139.9 (C-4a), 151.8 (C-3), 152.3 (C-1). Anal. Calcd for $\mathrm{C}_{15} \mathrm{H}_{11} \mathrm{ClN}_{2}$ (245.71): C, 70.73; H, 4.35; N, 11.00\%. Found: C, 70.63; H, $4.54 ; \mathrm{N}, 10.71 \%$.

$\mathrm{N}$-(2-Bromophenyl)isoquinolin-3-amine (2h). Reaction time $8 \mathrm{~h}$. Eluent: $\mathrm{CH}_{2} \mathrm{Cl}_{2}$; yield: $47 \%$; green crystals; $\mathrm{mp} 100-102{ }^{\circ} \mathrm{C} ; v_{\max }\left(\mathrm{KBr}, \mathrm{cm}^{-1}\right): 3390,1588,1484,1304,754 ; \delta_{\mathrm{H}}(300 \mathrm{MHz}$, $\mathrm{CDCl}_{3}$ ): 6.84-6.96 (2H, m, NH, H-5'), 7.20-7.38 (3H, m, H-4, H-4', H-7), 7.52-7.65 (3H, m, H-6, H-3', H-6'), 7.76-7.88 (2H, m, H-5, H-8), 9.00 (1H, s, H-1); $\delta_{\mathrm{C}}\left(75 \mathrm{MHz}, \mathrm{CDCl}_{3}\right): 101.5$ (C-4), 114.6 (C-2'), 119.3 (C-4'), 123.1 (C-5), 124.5 (C-7), 125.3 (C-8a), 125.6 (C-6'), 128.0 (C-8), 128.4 (C-5'), 130.9 (C-6), 133.3 (C-3'), 138.6 (C-4a), 139.4 (C-1'), 151.0 (C-3), 152.3 (C-1). Anal. Calcd for $\mathrm{C}_{15} \mathrm{H}_{11} \mathrm{BrN}_{2}$ (299.16): C, 60.22; H, 3.71; N, 9.36\%. Found: C, 60.49; H, 3.79; N, $9.07 \%$.

$\mathbf{N}$-(3-Bromophenyl)isoquinolin-3-amine (2i). Reaction time $1 \mathrm{~h}$. Eluent: Hexane /EtOAc (4:1); yield: $46 \%$; greenish yellow crystals; $\mathrm{mp} 122-125^{\circ} \mathrm{C}$; $v_{\max }\left(\mathrm{KBr}, \mathrm{cm}^{-1}\right): 2925,2853,1589,1361$, 747; $\delta_{\mathrm{H}}\left(300 \mathrm{MHz}, \mathrm{CDCl}_{3}\right): 6.78(1 \mathrm{H}, \mathrm{s}, \mathrm{NH}), 7.14-7.25\left(4 \mathrm{H}, \mathrm{m}, \mathrm{NH}, \mathrm{H}-2^{\prime}, \mathrm{H}^{-} 4^{\prime}, \mathrm{H}-5^{\prime}\right), 7.34(1 \mathrm{H}$, $\mathrm{dd}, J=7.4 \mathrm{~Hz}, 7.4 \mathrm{~Hz}, \mathrm{H}-7), 7.53-7.63$ (3H, m, H-6', H-6, H-8), 7.84 (1H, d, $J=8.1 \mathrm{~Hz}, \mathrm{H}-5)$, $8.97(1 \mathrm{H}, \mathrm{s}, \mathrm{H}-1) ; \delta_{\mathrm{C}}\left(75 \mathrm{MHz}, \mathrm{CDCl}_{3}\right): 100.5(\mathrm{C}-4), 118.0\left(\mathrm{C}-2^{\prime}\right), 122.1\left(\mathrm{C}-6^{\prime}\right), 123.3\left(\mathrm{C}-3^{\prime}\right)$, 124.4 (C-4'), 125.1 (C-8a), 125.3 (C-7), 125.6 (C-5), 127.9 (C-8), 130.9 (C-6), 131.0 (C-5'), 138.7 (C-4a), 142.8 (C-1'), 151.2 (C-3), 152.3 (C-1). Anal. Calcd for $\mathrm{C}_{15} \mathrm{H}_{11} \mathrm{BrN}_{2}$ (299.16): C, 60.22; H, 3.71; N, 9.36\%. Found: C, 60.31; H, 3.82; N, 9.45\%.

4-Benzyl- $\boldsymbol{N}$-(3-bromophenyl)isoquinolin-3-amine (2j). Reaction time $1 \mathrm{~h}$. Eluent: Hexane /EtOAc (3:1); yield: 49\%; yellow crystals; mp 60-62 ${ }^{\circ} \mathrm{C} ; v_{\max }\left(\mathrm{KBr}, \mathrm{cm}^{-1}\right): 1591,1572,1489$, 994, 751; $\delta_{\mathrm{H}}\left(300 \mathrm{MHz}, \mathrm{CDCl}_{3}\right): 4.39\left(2 \mathrm{H}, \mathrm{s}, \mathrm{CH}_{2}\right), 6.17(1 \mathrm{H}, \mathrm{s}, \mathrm{NH}), 7.04-7.33\left(8 \mathrm{H}, \mathrm{m}, \mathrm{H}-2^{\prime}, \mathrm{H}-\right.$ 4', H-5', H-2", H-3", H-4", H-5", H-6"), 7.40-7.47 (2H, m, H-7, H-6'), 7.64 (1H, dd, J= 7.9, 7.6 $\mathrm{Hz}, \mathrm{H}-6), 7.91-7.95$ (2H, m, H-5, H-8), $9.05(1 \mathrm{H}, \mathrm{s}, \mathrm{H}-1)$; $\delta_{\mathrm{C}}\left(75 \mathrm{MHz}, \mathrm{CDCl}_{3}\right): 32.1\left(\mathrm{CH}_{2}\right)$, 112.7 (C-4), 117.1 (C-2'), 121.2 (C-6'), 122.5 (C-4'), 122.9 (C-3'), 124.2 (C-5), 124.4 (C-7), 125.7 (C-8a), 127.2 (C-4"), 128.3 (C-2", C-6"), 128.8 (C-8), 129.4 (C-3", C-5"), 130.3 (C-6), $131.1\left(\mathrm{C}-5^{\prime}\right), 137.7$ (C-4a), $138.4\left(\mathrm{C}-1^{\prime}\right), 143.6\left(\mathrm{C}-1^{\prime}\right), 149.0$ (C-3), 150.6 (C-1). Anal. Calcd for $\mathrm{C}_{22} \mathrm{H}_{17} \mathrm{BrN}_{2}$ (389.28): C, 67.88; H, 4.40; N, 7.20\%. Found: C, 67.65; H, 4.53; N, 7.31\%.

$\mathrm{N}$-(4-Bromophenyl)isoquinolin-3-amine (2k). Reaction time 8 h. Eluent: $\mathrm{CH}_{2} \mathrm{Cl}_{2}$; yield: $80 \%$, yellow crystals; mp $159-162{ }^{\circ} \mathrm{C} ; v_{\max }\left(\mathrm{KBr}, \mathrm{cm}^{-1}\right): 3269,3050,1631,1580,749 ; \delta_{\mathrm{H}}(300 \mathrm{MHz}$, $\left.\mathrm{CDCl}_{3}\right): 6.90$ (1H, s, H-4), 7.05-7.19 (5H, m, H-2', H-3', H-5', H-6', H-7), 7.29-7.37 (2H, m, H6, H-5), $7.58(1 \mathrm{H}, \mathrm{d}, J=8.1 \mathrm{~Hz}, \mathrm{H}-8), 7.69(1 \mathrm{H}, \mathrm{s}, \mathrm{NH}), 8.72(1 \mathrm{H}, \mathrm{s}, \mathrm{H}-1) ; \delta_{\mathrm{C}}\left(75 \mathrm{MHz}, \mathrm{CDCl}_{3}\right)$ : 100.6 (C-4), 113.2 (C-4'), 120.6 (C-2', C-6'), 123.8 (C-5), 124.6 (C-8a), 125.3 (C-7), 127.7 (C8), 130.6 (C-6), 131.9 (C-3', C-5'), 138.5 (C-4a), 141.1 (C-1'), 151.6 (C-1), 151.8 (C-3). Anal. Calcd for $\mathrm{C}_{15} \mathrm{H}_{11} \mathrm{BrN}_{2}$ (299.16): C, 60.22; H, 3.71; N, 9.36\%. Found: C, 60.53; H, 3.76; N, $9.11 \%$. 
$\mathrm{N}$-(2-Nitrophenyl)isoquinolin-3-amine (2l). Reaction time $5 \mathrm{~h}$. Eluent: $\mathrm{CH}_{2} \mathrm{Cl}_{2}$; yield: $94 \%$; dark orange crystals; mp, $148-149{ }^{\circ} \mathrm{C} ; v_{\max }\left(\mathrm{KBr}, \mathrm{cm}^{-1}\right): 3340,1617,1509,1263,732 ; \delta_{\mathrm{H}}(300$ $\mathrm{MHz}_{\mathrm{CDCl}}$ ): 6.93 (1H, dd, $\left.J=7.7 \mathrm{~Hz}, 8.4 \mathrm{~Hz}, \mathrm{H}-4^{\prime}\right), 7.39$ (1H, s, H-4), 7.45-7.56 (2H, m, H-5', H-7), 7.62-7.75 (2H, m, H-6, H-8), 7.93 (1H, dd, $J=8.2$ Hz, H-5), 8.27-8.29 (2H, m, H-3', H-6'), $9.10(1 \mathrm{H}, \mathrm{s}, \mathrm{H}-1), 10.14(1 \mathrm{H}, \mathrm{s}, \mathrm{NH}) ; \delta_{\mathrm{C}}\left(75 \mathrm{MHz}, \mathrm{CDCl}_{3}\right): 107.9(\mathrm{C}-4), 118.7\left(\mathrm{C}-6^{\prime}\right), 119.4(\mathrm{C}-$ 4'), 125.9 (C-7), 126.0 (C-5), 126.2 (C-8a), 126.7 (C-3'), 128.0 (C-8), 131.2 (C-6), 134.9 (C-2'), $135.8\left(\mathrm{C}-5^{\prime}\right), 138.3$ (C-4a), 140.1 (C-1'), 148.9 (C-3), 152.1 (C-1). Anal. Calcd for $\mathrm{C}_{15} \mathrm{H}_{11} \mathrm{~N}_{3} \mathrm{O}_{2}$ (265.26): C, 67.92; H, 4.18; N, 15.84\%. Found: C, 67.99; H, 4.09; N, 15.78\%.

4-Methyl- $\mathbf{N}$-(2-nitrophenyl)isoquinolin-3-amine (2m). Reaction time 5 h. Eluent: $\mathrm{CH}_{2} \mathrm{Cl}_{2}$; yield: $94 \%$; dark orange crystals; $\mathrm{mp} 130-133{ }^{\circ} \mathrm{C} ; v_{\max }\left(\mathrm{KBr}, \mathrm{cm}^{-1}\right)$ : 3341, 1616, 1509, 1249, 734; $\delta_{\mathrm{H}}\left(300 \mathrm{MHz}, \mathrm{CDCl}_{3}\right): 2.64\left(3 \mathrm{H}, \mathrm{s}, \mathrm{CH}_{3}\right), 6.87\left(1 \mathrm{H}, \mathrm{dd}, J=7.4 \mathrm{~Hz}, 8.1 \mathrm{~Hz}, \mathrm{H}-44^{\prime}\right), 7.45-7.55(2 \mathrm{H}$, m, H-5', H-7), 7.72 (1H, dd, $J=7.4 \mathrm{~Hz}, 8.0 \mathrm{~Hz}, \mathrm{H}-6), 7.94-8.04$ (3H, m, H-5, H-8, H-5'), 8.24 $\left(1 \mathrm{H}, \mathrm{d}, J=8.6 \mathrm{~Hz}, \mathrm{H}-3^{\prime}\right), 9.02(1 \mathrm{H}, \mathrm{s}, \mathrm{H}-1), 10.2(1 \mathrm{H}, \mathrm{s}, \mathrm{NH}) ; \delta_{\mathrm{C}}\left(75 \mathrm{MHz}, \mathrm{CDCl}_{3}\right): 12.6\left(\mathrm{CH}_{3}\right)$, 116.4 (C-4), $118.7\left(\mathrm{C}-6^{\prime}\right), 118.8$ (C-4'), 123.2 (C-5), 125.8 (C-7), 126.5 (C-3'), 127.8 (C-4a), 128.5 (C-8), 130.9 (C-6), 134.2 (C-2'), 135.8 (C-5'), 137.4 (C-4a), 141.3 (C-1'), 146.3 (C-3), 149.7 (C-1). Anal. Calcd for $\mathrm{C}_{16} \mathrm{H}_{13} \mathrm{~N}_{3} \mathrm{O}_{2}$ (279.29): C, 68.81; H, 4.69; N, 15.05\%. Found: C, $68.62 ; \mathrm{H}, 4.80 ; \mathrm{N}, 14.83 \%$.

4-Benzyl- $N$-(2-nitrophenyl)isoquinolin-3-amine (2n). Reaction time $5 \mathrm{~h}$. Eluent: $\mathrm{CH}_{2} \mathrm{Cl}_{2}$; yield: $95 \%$; orange crystals; $\mathrm{mp} 143-146^{\circ} \mathrm{C} ; v_{\max }\left(\mathrm{KBr}, \mathrm{cm}^{-1}\right): 3365,1613,1495,1247,738 ; \delta_{\mathrm{H}}$ $\left(300 \mathrm{MHz}, \mathrm{CDCl}_{3}\right): 4.53\left(2 \mathrm{H}, \mathrm{s}, \mathrm{CH}_{2}\right), 6.88\left(1 \mathrm{H}, \mathrm{dd}, J=7.2,7.1 \mathrm{~Hz}, \mathrm{C}-4^{\prime}\right), 7.15-7.27$ (5H, m, H2", H-3", H-4", H-5", H-6"), 7.47-7.55 (2H, m, H-5', H-7), 7.71 (1H, dd, J = 8.4, 8.2 Hz, H-6), 7.97-8.03 (3H, m, H-8, H-5, H-6'), 8.20 (1H, d, $J=8.6$ Hz, H-3'), 9.11 (1H, s, H-1), 10.07 (1H, s, $\mathrm{NH}) ; \delta_{\mathrm{C}}\left(75 \mathrm{MHz}, \mathrm{CDCl}_{3}\right): 32.2\left(\mathrm{CH}_{2}\right), 118.6(\mathrm{C}-4), 118.7\left(\mathrm{C}-4{ }^{\prime}\right), 118.9\left(\mathrm{C}-6^{\prime}\right), 123.3(\mathrm{C}-5)$, 125.7 (C-7), 126.2 (C-3'), 126.6 (C-8a), 126.7 (C-4"), 128.1 (C-2", C-6"), 128.4 (C-8), 128.8 (C3", C-5"), 131.2 (C-6), 134.3 (C-2'), 135.3 (C-5'), 137.4 (C-4a), 137.9 (C-1"), 140.8 (C-1'), 146.8 (C-3), 150.4 (C-1). Anal. Calcd for $\mathrm{C}_{22} \mathrm{H}_{17} \mathrm{~N}_{3} \mathrm{O}_{2}$ (355.38): C, 74.35; H, 4.82; N, 11.82\%. Found: C, 74.52; H, 4.80; N, 11.95\%.

$\mathbf{N}$-(4-Nitrophenyl)isoquinolin-3-amine (2o). Reaction time 4 h. Eluent: Hexane /EtOAc (3:1); yield: 55\%; brownish yellow crystals; $\mathrm{mp} 174-176^{\circ} \mathrm{C} ; v_{\max }\left(\mathrm{KBr}, \mathrm{cm}^{-1}\right): 3229,1582,1495,1318$, $747 ; \delta_{\mathrm{H}}\left(300 \mathrm{MHz}, \mathrm{CDCl}_{3}\right): 7.31(1 \mathrm{H}, \mathrm{s}, \mathrm{H}-4), 7.40(1 \mathrm{H}, \mathrm{dd}, J=7.5,7.6 \mathrm{~Hz}, \mathrm{H}-7), 7.61(1 \mathrm{H}, \mathrm{dd}$, $J=7.6,7.5 \mathrm{~Hz}, \mathrm{H}-6), 7.70-7.75$ (3H, m, H-5, H-2', H-6'), 7.91 (1H, d, $J=7.4 \mathrm{~Hz}, \mathrm{H}-8), 8.12-$ $8.15\left(2 \mathrm{H}, \mathrm{m}, \mathrm{H}-3^{\prime}, \mathrm{H}-5^{\prime}\right), 9.06(1 \mathrm{H}, \mathrm{s}, \mathrm{H}-1), 9.57(1 \mathrm{H}, \mathrm{s}, \mathrm{NH}) ; \delta_{\mathrm{C}}\left(75 \mathrm{MHz}, \mathrm{CDCl}_{3}\right): 104.2(\mathrm{C}-4)$, 115.4 (C-2', C-6'), 124.2 (C-7), 124.5 (C-8a), 124.9 (C-3', C-5'), 125.0 (C-5), 127.2 (C-8), 130.2 (C-6), 137.5 (C-4a), 139.0 (C-4'), 148.5 (C-1'), 149.8 (C-3), 150.6 (C-1). Anal. Calcd for $\mathrm{C}_{15} \mathrm{H}_{11} \mathrm{~N}_{3} \mathrm{O}_{2}$ (265.26): C, 67.92; H, 4.18; N, 15.84\%. Found: C, 67.99; H, 4.03; N, 15.69\%.

4-Methyl- $\mathbf{N}$-(4-nitrophenyl)isoquinolin-3-amine (2p). Reaction time $2 \mathrm{~h}$. Eluent: $\mathrm{CH}_{3} \mathrm{Cl}$; yield: $60 \%$; brownish orange crystals; $\mathrm{mp} 196-199{ }^{\circ} \mathrm{C} ; v_{\max }\left(\mathrm{KBr}, \mathrm{cm}^{-1}\right): 3354,1603,1329,1114,743$; $\delta_{\mathrm{H}}\left(300 \mathrm{MHz}, \mathrm{CDCl}_{3}\right): 2.55\left(3 \mathrm{H}, \mathrm{s}, \mathrm{CH}_{3}\right), 6.76(1 \mathrm{H}, \mathrm{s}, \mathrm{NH}), 7.21-7.24\left(2 \mathrm{H}, \mathrm{m}, \mathrm{H}-2^{\prime}, \mathrm{H}-6^{\prime}\right), 7.52$ $(1 \mathrm{H}, \mathrm{dd}, J=7.6 \mathrm{~Hz}, 7.4 \mathrm{~Hz}, \mathrm{H}-7), 7.73(1 \mathrm{H}, \mathrm{dd}, J=7.4 \mathrm{~Hz}, 7.7 \mathrm{~Hz}, \mathrm{H}-6), 7.95-7.98$ (2H, m, H-8, H-5), 8.14-8.18 (2H, m, H-3', H-5'), 9.00 (1H, s, H-1); $\delta_{\mathrm{C}}\left(75 \mathrm{MHz}, \mathrm{CDCl}_{3}\right): 12.8\left(\mathrm{CH}_{3}\right), 114.9$ 
(C-4), 115.7 (C-2', C-6'), 123.1 (C-5), 125.7 (C-7), 126.0 (C-3', C-5'), 126.5 (C-8a), 128.6 (C-8), 131.0 (C-6), 137.4 (C-4a), 140.8 (C-4'), 146.4 (C-1'), 149.2 (C-3), 150.0 (C-1). Anal. Calcd for $\mathrm{C}_{16} \mathrm{H}_{13} \mathrm{~N}_{3} \mathrm{O}_{2}$ (279.29): C, 68.81; H, 4.69; N, 15.05\%. Found: C, 68.59; H: 4.78; N, 15.25\%.

4-Benzyl- $\mathbf{N}$-(4-nitrophenyl)isoquinolin-3-amine (2q). Reaction time $2 \mathrm{~h}$. Eluent: $\mathrm{CH}_{2} \mathrm{Cl}_{2}$; yield: $64 \%$; brownish yellow crystals; $\mathrm{mp} 120-122{ }^{\circ} \mathrm{C} ; v_{\max }\left(\mathrm{KBr}, \mathrm{cm}^{-1}\right): 3426,1588,1310,1113$, 747; $\delta_{\mathrm{H}}\left(300 \mathrm{MHz}, \mathrm{CDCl}_{3}\right): 4.44\left(2 \mathrm{H}, \mathrm{s}, \mathrm{CH}_{2}\right), 6.65(1 \mathrm{H}, \mathrm{s}, \mathrm{NH}), 7.16-7.34\left(7 \mathrm{H}, \mathrm{m}, \mathrm{H}-2^{\prime}, \mathrm{H}-6^{\prime}\right.$, H-2", H-3", H-4", H-5", H-6"), 7.51 (1H, dd, J=7.2 Hz, 7.8 Hz, H-7), 7.70 (1H, dd, J= 7.8, 8.0 Hz, H-6), 7.98-8.01 (2H, m, H-5, H-8), 8.11-8.13 (2H, m, H-3', H-5'), 9.08 (1H, s, H-1); $\delta_{\mathrm{C}}(75$ $\left.\mathrm{MHz}, \mathrm{CDCl}_{3}\right): 32.2\left(\mathrm{CH}_{2}\right), 115.7$ (C-4), 116.4 (C-2', C-6'), 122.9 (C-5), 125.5 (C-7), 125.8 (C-3', C-5'), 126.4 (C-8a), 127.4 (C-4"), 128.3 (C-2", C-6"), 128.8 (C-8), 129.5 (C-3", C-5"), 131.5 (C6), 137.5 (C-4a), 138.1 (C-1"), 140.8 (C-4'), 147.5 (C-1'), 148.5 (C-3), 150.7 (C-1). Anal. Calcd for $\mathrm{C}_{22} \mathrm{H}_{17} \mathrm{~N}_{3} \mathrm{O}_{2}$ (355.38): C, 74.35; H, 4.82; N, 11.82\%. Found: C, 74.51; H, 4.75; N, 11.61\%.

Ethyl 2-(isoquinolin-3-ylamino)benzoate (2r). Reaction time $6 \mathrm{~h}$. Eluent: Hexane /EtOAc (3:1); yield: $43 \%$, green crystals; $m p 44-46{ }^{\circ} \mathrm{C} ; v_{\max }\left(\mathrm{KBr}, \mathrm{cm}^{-1}\right): 3298,3049,1614,1451,745$; $\delta_{\mathrm{H}}\left(300 \mathrm{MHz}, \mathrm{CDCl}_{3}\right): 1.42\left(3 \mathrm{H}, \mathrm{t}, J=7.1,7.1 \mathrm{~Hz}, \mathrm{CH}_{3}\right), 4.39\left(2 \mathrm{H}, \mathrm{q}, J=7.1,14.28 \mathrm{~Hz}, \mathrm{CH}_{2}\right)$, $6.88\left(1 \mathrm{H}, \mathrm{dd}, J=7.2,7.9 \mathrm{~Hz}, \mathrm{H}-5^{\prime}\right), 7.38\left(1 \mathrm{H}, \mathrm{dd}, J=7.0,7.8 \mathrm{~Hz}, \mathrm{H}-4^{\prime}\right), 7.47(1 \mathrm{H}, \mathrm{dd}, J=7.7$, $7.8 \mathrm{~Hz}, \mathrm{H}-7), 7.55$ (1H, dd, $J=7.8,7.2 \mathrm{~Hz}, \mathrm{H}-6), 7.60-7.62(2 \mathrm{H}, \mathrm{m}, \mathrm{H}-5, \mathrm{H}-4), 7.85(1 \mathrm{H}, \mathrm{d}, J=$ $8.1 \mathrm{~Hz}, \mathrm{H}-8), 8.05$ (1H, d, $\left.J=8.0 \mathrm{~Hz}, \mathrm{H}-3^{\prime}\right), 8.26\left(1 \mathrm{H}, \mathrm{d}, J=8.3 \mathrm{~Hz}, \mathrm{H}-6^{\prime}\right), 9.04$ (1H, s, H-1), $10.42(1 \mathrm{H}, \mathrm{s}, \mathrm{NH}) ; \delta_{\mathrm{C}}\left(75 \mathrm{MHz}, \mathrm{CDCl}_{3}\right): 14.5\left(\mathrm{CH}_{3}\right), 61.1\left(\mathrm{CH}_{2}\right), 105.0(\mathrm{C}-4), 113.9\left(\mathrm{C}-1^{\prime}\right)$, $117.0\left(\mathrm{C}-3^{\prime}\right), 119.0\left(\mathrm{C}-5^{\prime}\right), 124.7$ (C-7), 125.4 (C-8a), 125.7 (C-5), 127.9 (C-8), 130.7 (C-6'), 131.6 (C-6), 134.2 (C-4'), 138.5 (C-4a), 145.5 (C-2'), 150.7 (C-3), 151.7 (C-1), $168.7(\mathrm{C}=\mathrm{O})$. Anal. Calcd for $\mathrm{C}_{18} \mathrm{H}_{16} \mathrm{~N}_{2} \mathrm{O}_{2}$ (292.33): C, 73.95; H, 5.52; N, 9.58\%. Found: C, 74.06; H, 5.72; N, $9.35 \%$.

Ethyl 3-(isoquinolin-3-ylamino)benzoate (2s). Reaction time $6 \mathrm{~h}$. Eluent: Hexane /EtOAc (3:1); yield: 84\%; yellow crystals; $\mathrm{mp} 107-109{ }^{\circ} \mathrm{C} ; v_{\max }\left(\mathrm{KBr}, \mathrm{cm}^{-1}\right): 3257,3060,1721,1365$, 745; $\delta_{\mathrm{H}}\left(300 \mathrm{MHz}, \mathrm{CDCl}_{3}\right): 1.40\left(3 \mathrm{H}, \mathrm{t}, J=6.9,7.2 \mathrm{~Hz}, \mathrm{CH}_{3}\right), 4.39(2 \mathrm{H}, \mathrm{q}, J=7.2,14.1 \mathrm{~Hz}$, $\left.\mathrm{CH}_{2}\right), 6.79(1 \mathrm{H}, \mathrm{s}, \mathrm{NH}), 7.19(1 \mathrm{H}, \mathrm{s}, \mathrm{H}-4), 7.33(1 \mathrm{H}, \mathrm{dd}, J=7.2,7.2 \mathrm{H}-7), 7.42(1 \mathrm{H}, \mathrm{dd}, J=7.8$, $\left.7.5 \mathrm{~Hz}, \mathrm{H}-5^{\prime}\right), 7.52-7-59$ (3H, m, H-5, H-6, H-4'), 7.72 (1H, d, J=7.2 Hz, H-6'), 7.83 (1H, d, $J=$ $7.8 \mathrm{~Hz}, \mathrm{H}-8), 7.99\left(1 \mathrm{H}, \mathrm{s}, \mathrm{H}-2^{\prime}\right), 8.98(1 \mathrm{H}, \mathrm{s}, \mathrm{H}-1)$; $\delta_{\mathrm{C}}\left(75 \mathrm{MHz}, \mathrm{CDCl}_{3}\right): 14.6\left(\mathrm{CH}_{3}\right), 61.3\left(\mathrm{CH}_{2}\right)$, 100.1 (C-4), 120.6 (C-2'), 123.6 (C-6'), 123.9 (C-5), 124.2 (C-7), 125.0 (C-8a), 125.5 (C-4'), 128.0 (C-8), $129.6\left(\mathrm{C}^{\prime} 5^{\prime}\right), 130.9$ (C-6), 132.0 (C-1'), 138.7 (C-4a), 141.5 (C-3'), 151.6 (C-3), $152.3(\mathrm{C}-1), 166.7(\mathrm{C}=\mathrm{O})$. Anal. Calcd for $\mathrm{C}_{18} \mathrm{H}_{16} \mathrm{~N}_{2} \mathrm{O}_{2}$ (292.33): C, 73.95; H, 5.52; N, 9.58\%. Found: C, 74.01; H, 5.30; N, 9.63\%.

Ethyl 4-(isoquinolin-3-ylamino)benzoate (2t). Reaction time 6 h. Eluent: Hexane /EtOAc (4:1); yield: $83 \%$; yellow crystals; mp $141-144{ }^{\circ} \mathrm{C} ; v_{\max }\left(\mathrm{Br}, \mathrm{cm}^{-1}\right): 3357,2977,1679,1285,770 ; \delta_{\mathrm{H}}$ $\left(300 \mathrm{MHz}, \mathrm{CDCl}_{3}\right): 1.39\left(3 \mathrm{H}, \mathrm{m}, \mathrm{CH}_{3}\right), 4.38\left(2 \mathrm{H}, \mathrm{m}, \mathrm{CH}_{2}\right), 7.09$ (1H. s, NH), 7.34-7.37 (4H, m, H-2', H-6', H-4, H-7), 7.60-7.64 (2H, m, H-6, H-5), 7.86 (1H, d, J = 7.1 Hz, H-8), 8.02- 8.05 $\left(2 \mathrm{H}, \mathrm{m}, \mathrm{H}-3^{\prime}, \mathrm{H}-5^{\prime}\right), 9.00(1 \mathrm{H}, \mathrm{s}, \mathrm{H}-1)$; $\delta_{\mathrm{C}}\left(75 \mathrm{MHz}, \mathrm{CDCl}_{3}\right): 14.7\left(\mathrm{CH}_{3}\right), 60.8\left(\mathrm{CH}_{2}\right), 102.1(\mathrm{C}-$ 4), 117.0 (C-3', C-5'), 123.3 (C-1'), 124.7 (C-7), 125.3 (C-8a), 125.7 (C-5), 127.9 (C-8), 131.1 (C-6), 131.5 (C-2', C-6'), 138.5 (C-4a), 145.8 (C-4'), 150.3 (C-3), 152.3 (C-1), $166.6(\mathrm{C}=\mathrm{O})$. 
Anal. Calcd for $\mathrm{C}_{18} \mathrm{H}_{16} \mathrm{~N}_{2} \mathrm{O}_{2}$ (292.33): C, 73.95; H, 5.52; N, 9.58\%. Found: C, 73.61; H, 5.52; N, $9.67 \%$.

2-(Isoquinolin-3-ylamino)benzaldehyde (2u). Reaction time $2 \mathrm{~h}$. Eluent: Hexane /EtOAc (2:1); yield: 84\%; brownish yellow; mp 92-95 ${ }^{\circ} \mathrm{C} ; v_{\max }\left(\mathrm{KBr}, \mathrm{cm}^{-1}\right): 2819,2741,1602,1447,754 ; \delta_{\mathrm{H}}$ $\left(300 \mathrm{MHz}, \mathrm{CDCl}_{3}\right): 6.98\left(1 \mathrm{H}, \mathrm{dd}, J=7.3,7.3 \mathrm{~Hz}, \mathrm{H}-5^{\prime}\right), 7.31(1 \mathrm{H}, \mathrm{s}, \mathrm{H}-4), 7.41(1 \mathrm{H}, \mathrm{dd}, J=7.3$, 7.4, H-7), 7.54-7.71 (4H, m, H-5, H-6, H-4', H-6'), 7.89 (1H, d, $J=8.1 \mathrm{~Hz}, \mathrm{H}-8), 8.45$ (1H, d, $J=$ $\left.8.4 \mathrm{~Hz}, \mathrm{H}-3^{\prime}\right), 9.08(1 \mathrm{H}, \mathrm{s}, \mathrm{H}-1), 9.95(1 \mathrm{H}, \mathrm{s}, \mathrm{CHO}), 10.90(1 \mathrm{H}, \mathrm{s}, \mathrm{NH}) ; \delta_{\mathrm{C}}\left(75 \mathrm{MHz}, \mathrm{CDCl}_{3}\right)$ : 106.4 (C-4), 116.3 (C-3'), 119.0 (C-5'), 120.5 (C-1'), 125.1 (C-7), 125.6 (C-8a), 125.8 (C-5), 127.9 (C-8), 130.9 (C-6), 136.0 (C-4'), 136.8 (C-6'), 138.4 (C-4a), 145.6 (C-2'), 150.1 (C-3), 151.6 (C-1), 194.9 (CHO). Anal. Calcd for $\mathrm{C}_{16} \mathrm{H}_{12} \mathrm{~N}_{2} \mathrm{O}$ (248.27): C, 77.40; H, 4.87; N, 11.28\%. Found: C, 77.25; H, 4.82; N, 11.41\%.

$\mathrm{N}$-(3-Bromopyridin-2-yl)isoquinolin-3-amine (2v). Reaction time $10 \mathrm{~h}$. Eluent: Hexane /EtOAc (2:1); yield: 80\%; brownish yellow; mp 107-110 ${ }^{\circ} \mathrm{C} ; v_{\max }\left(\mathrm{KBr}, \mathrm{cm}^{-1}\right): 3386,1584,1512$, 1014,$742 ; \delta_{\mathrm{H}}\left(300 \mathrm{MHz}, \mathrm{CDCl}_{3}\right): 6.72(1 \mathrm{H}, \mathrm{m}, \mathrm{H}-5$ '), $7.39(1 \mathrm{H}, \mathrm{dd}, J=7.8,7.3 \mathrm{~Hz}, \mathrm{H}-7), 7.59$ $(1 \mathrm{H}, \mathrm{dd}, J=8.1,7.1 \mathrm{~Hz}, \mathrm{H}-6), 7.78-7.87$ (3H, m, H-4', H-5, H-8), 7.99 (1H, s, NH), $8.31(1 \mathrm{H}, \mathrm{d}$, $\left.J=4.7 \mathrm{~Hz}, \mathrm{H}-6^{\prime}\right), 8.78(1 \mathrm{H}, \mathrm{s}, \mathrm{H}-4), 8.98(1 \mathrm{H}, \mathrm{s}, \mathrm{H}-1) ; \delta_{\mathrm{C}}\left(75 \mathrm{MHz}, \mathrm{CDCl}_{3}\right): 105.3(\mathrm{C}-4), 107.2$ (C-3'), 116.5 (C-5'), 124.9 (C-7), 125.9 (C-8a), 126.8 (C-5), 127.7 (C-8), 130.7 (C-6), 138.5 (C4a), 140.5 (C-4'), 146.5 (C-6'), 148.4 (C-3), 151.1 (C-2'), 151.3 (C-1). Anal. Calcd for $\mathrm{C}_{14} \mathrm{H}_{10} \mathrm{BrN}_{3}$ (300.15): C, 56.02; H, 3.36; N, 14.00\%. Found: C, 56.06; H, 3.48; N,13.88\%.

4-Benzyl- $\boldsymbol{N}$-(3-bromopyridin-2-yl)isoquinolin-3-amine (2w). Reaction time $10 \mathrm{~h}$. Eluent: Hexane /EtOAc (2:1); yield: 70\%; brownish yellow crystals; mp 116-118 ${ }^{\circ} \mathrm{C} ; v_{\max }\left(\mathrm{KBr}, \mathrm{cm}^{-1}\right)$ : 3396, 1590, 1488, 1012, 728; $\delta_{\mathrm{H}}\left(300 \mathrm{MHz}, \mathrm{CDCl}_{3}\right): 4.45\left(2 \mathrm{H}, \mathrm{s}, \mathrm{CH}_{2}\right), 6.64\left(1 \mathrm{H}, \mathrm{m}, \mathrm{H}-5^{\prime}\right), 7.03$ $(1 \mathrm{H}, \mathrm{s}, \mathrm{NH}), 7.15-7.25$ (5H, m, H-2", H-3", H-4", H-5", H-6"), 7.52 (1H, dd, J = 7.6, 7.5 Hz, H7), 7.65 (1H, dd, $J=7.8,8.0 \mathrm{~Hz}, \mathrm{H}-6), 7.73(1 \mathrm{H}, \mathrm{d}, J=7.8 \mathrm{~Hz}, \mathrm{H}-4$ '), 7.97-8.02 (2H, m, H-5, H8), $8.1\left(1 \mathrm{H}, \mathrm{d}, J=4.7 \mathrm{~Hz}, \mathrm{H}-6{ }^{\prime}\right), 9.16(1 \mathrm{H}, \mathrm{s}, \mathrm{H}-1)$; $\delta_{\mathrm{C}}\left(75 \mathrm{MHz}, \mathrm{CDCl}_{3}\right): 33.1\left(\mathrm{CH}_{2}\right), 107.2(\mathrm{C}-$ 3'), 116.6 (C-5'), 122.2 (C-4), 123.7 (C-5), 126.0 (C-7), 126.6 (C-4"), 127.5 (C8a), 128.5 (C-3", C-5"), 128.5 (C-8), 128.9 (C-2", C-6"), 130.9 (C-6), 137.4 (C-4a), 139.4 (C-1"), 140.5 (C-4'), 146.7 (C-3), 147.1 (C-6'), 151.2 (C-1), 153.1 (C-2'). Anal. Calcd for $\mathrm{C}_{21} \mathrm{H}_{16} \mathrm{BrN}_{3}$ (390,27): C, 64.63; H, 4.13; N, 10.77\%. Found: C, 64.91; H, 4.21; N: 10.39\%.

\section{Acknowledgements}

The support of OTKA 77784, K75015, Nanotransport (CRC-HAS-2009) and Hungarian GVOP3.2.1.-2004-04-0210/3.0 projects are gratefully acknowledged. 


\section{References}

1. Balog, J.; Riedl, Zs.; Hajós, Gy.; Miskolczy, Zs.; Biczók, L. Tetrahedron Lett. 2011, 53, 5264.

2. Guram, A. S.; Rennels, R. A.; Buchwald, S. L. Angew. Chem., Int. Ed. 1995, 34, 1348.

3. Louie, J.; Hartwig, J. F. Tetrahedron Lett. 1995, 36, 3609.

4. (a) Muci, A. R.; Buchwald, S. L. Top. Curr. Chem. 2002, 219, 131. (b) Schlummer, B.; Scholz, U. Adv. Synth. Catal. 2004, 346, 1599.

5. Van Baelen, G.; Meyers, C.; Lemiere, Guy L. F.; Hostyn, S.; Dommisse, R.; Maes, Bert U. W.; Maes, L.; Augustyns, K.; Haemers, A.; Pieters, L. Tetrahedron 2008, 64, 11802.

6. (a) Loones, K. T. J.; Maes, B. U. W.; Meyers, C.; Deruytter, J. J. Org. Chem. 2006 , 71, 260.

(b) Hostyn, S.; Maes, B. U. W.; Pieters, L.; Lemiére, G. L. F.; Mátyus, P.; Hajós, G.; Dommisse, R. A. Tetrahedron 2005, 61, 1571. (c) Hostyn, S.; Maes, B. U. W.; Van Baelen, G.; Gulevskaya, A.; Meyers, C.; Smits, K. Tetrahedron 2006, 62, 4676. (d) Jonckers, T. H. M.; Maes, B. U. W.; Lemiére, G. L. F.; Rombouts, G.; Pieters, L.; Haemers, A.; Dommisse, R.A. Synlett 2003, 615.

7. Megyesi, M.; Biczók, L.; Jablonkai, I. J. Phys. Chem. C 2008, 112, 3410.

8. Melhuish, W. H. J. Phys. Chem. 1961, 65, 229. 